\title{
On the Approximability of the Minimum Subgraph Diameter Problem
}

\author{
Arthur Pratti Dadalto ${ }^{1}$, Fábio Luiz Usberti ${ }^{1}$, Mário César San Felice ${ }^{2}$ \\ ${ }^{1}$ Instituto de Computacão - Universidade Estadual de Campinas (Unicamp) \\ Av. Albert Einstein, 1251 - Cidade Universitária - 13083-852 - Campinas - SP - Brazil \\ ${ }^{2}$ Departamento de Computação - Universidade Federal de São Carlos (UFSCar) \\ Rod. Washington Luís, Km 235 - 13565-905 - São Carlos - SP - Brazil
}

ra166858@students.ic.unicamp.br, fusbertidic.unicamp.br, felicedufscar.br

\begin{abstract}
This work addresses the minimum subgraph diameter problem $(M S D P)$ by answering an open question with respect to its approximability. Given a graph with lengths and costs associated to its edges, the MSDP consists in finding a spanning subgraph with total cost limited by a given budget, such that its diameter is minimum. We prove that there is no $\beta$-approximation algorithm for the MSDP, for any constant $\beta$, unless $P=N P$. Our proof is grounded on the non-approximability of the minimum spanning tree diameter problem, proven by Bálint in 2013.
\end{abstract}

\section{Introduction}

In a combinatorial optimization problem, there is a set of instances, a finite set of feasible solutions for each instance and an objective function. A feasible solution is one that respects a set of restrictions associated with the problem.

The problem can be of minimization or maximization. In a minimization problem, the goal is to find a feasible solution such that its value, evaluated by the objective function, is minimal. Such a solution is called optimal.

A naive algorithm to solve a combinatorial optimization problem consists in evaluating all feasible solutions in search of an optimal one. This easily becomes impractical, since many problems have an exponential amount of feasible solutions. While there are combinatorial optimization problems for which polynomial time algorithms are known, many of them are NP-hard problems for which there is no polynomial time algorithm, unless $P=N P$ [Papadimitriou and Steiglitz 1998].

For those problems, arises a need for approximation algorithms, which find feasible solutions in polynomial time to NP-hard optimization problems with provable guarantees on the distance between the returned solution and the optimal ones [Williamson and Shmoys 2011]. For a given constant $\beta$, we say that an algorithm is a $\beta$-approximation if, on every input, the algorithm finds a solution whose cost is at most $\beta$ times the optimum. However, for some problems, it is possible to prove that even the design of a $\beta$-approximate algorithm is impossible for small values of $\beta$ or even for any constant value of $\beta$, unless $P=N P$ [Trevisan 2014].

This paper studies the approximability of the minimum subgraph diameter problem (MSDP). Given a graph with lengths and costs associated to its edges, the MSDP 
consists in finding a spanning subgraph with total cost limited by a given budget, such that its diameter is minimum.

The MSDP can appear in real world applications, such as the construction of a telecommunication network with a budget constraint. There are costs related to building connections, e.g. acquisition of cables, and there are transmission delays between nodes inherent to the network topology. A relevant goal could be to minimize the largest delay between two nodes in the resulting network.

Our contribution. We prove that there is no polynomial time $\beta$-approximation algorithm for the MSDP for any constant $\beta$, unless $P=N P$.

Paper organization. Section 2 formally describes the MSDP. Section 3 explains previous results on the MSDP as well as related work. Section 4 shows the result obtained and Section 5 presents our future research directions.

\section{Problem description}

In this paper we study the minimum subgraph diameter problem (MSDP). The problem input consists of a graph $G=(V, E)$, a resource budget $R \in \mathbb{R}_{+}$and two weight functions for the edges: the length $l: E \rightarrow \mathbb{R}_{+}$and the cost $c: E \rightarrow \mathbb{R}_{+}$.

Given a spanning subgraph $S=\left(V, E^{\prime}\right)$ of $G$ and two vertices $u, v \in V$, we define $d_{s}(u, v)$ as the length of the shortest path between $u$ and $v$ in $S$ (using the $l$ weight function). If there is no path between vertices $u$ and $v$, then $d_{s}(u, v)=\infty$. The diameter of the subgraph is defined as $\operatorname{diam}(S)=\max _{u, v \in V} d_{s}(u, v)$. The cost of the subgraph is defined as $\operatorname{cost}(S)=\sum_{e \in E^{\prime}} c(e)$.

A feasible solution to this problem is a spanning subgraph $S$ with $\operatorname{cost}(S)$ at most $R$ and the goal is to minimize $\operatorname{diam}(S)$.

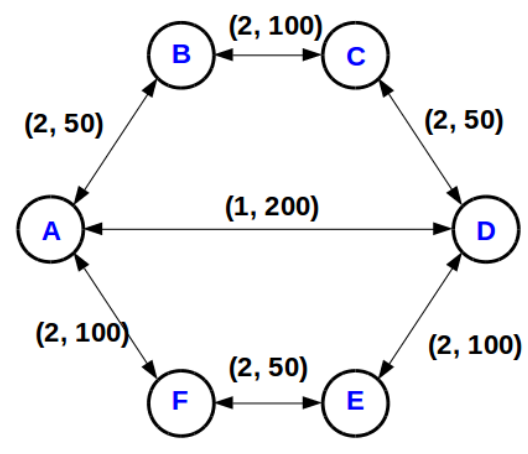

(a) MSDP instance.

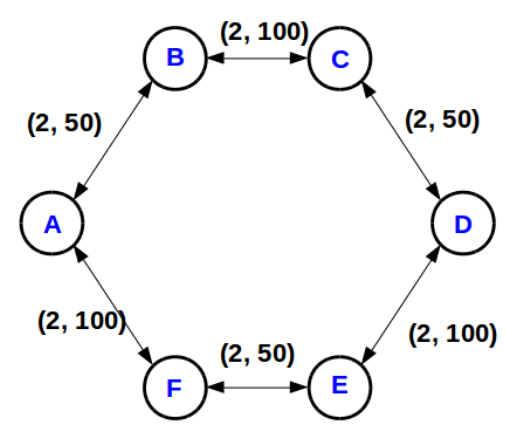

(b) An optimal solution.

Figure 1. Sample instance for the MSDP and an optimal solution for it.

Figure 1(a) shows a possible instance to the MSDP. Each edge is associated with a pair of values: the left one is its length and the right one is its cost. For feasible solutions a total cost budget of 450 will be considered. 
When trying to minimize the diameter one would not remove any edges, but the budget constraint does not allow it. When trying to minimize the total cost one would get to the minimum spanning tree, that does not minimize the diameter. An optimal solution for this instance is shown in Figure 1(b). It has a higher diameter than the original graph, but it is the lowest possible without violating the budget.

Let diam* be the diameter value for an optimal solution of the MSDP. Given a constant $\beta$, a $\beta$-approximation for the MSDP consists in finding a spanning subgraph $S$ such that $\operatorname{cost}(S) \leq R$ and $\operatorname{diam}(S) \leq \beta \cdot \operatorname{diam} *$.

\section{Related work}

It has been proved that, unless $P=N P$, there is no polynomial time $\beta$-approximation algorithm for the MSDP in each of the following four cases [Plesnik 1981]:

1. All the edge lengths (but not costs) of $G$ are equal and $\beta<5 / 4$.

2. $l(e)=c(e)$ for every edge $e$ and $\beta<5 / 4$.

3. $\beta<2$.

4. $G$ is a planar graph with maximum degree 3 and $\beta<3 / 2$.

It is worth mentioning that our work strengthens the result given in item 3 .

A more generic bicriteria network problem, the $(A, B, \lambda, \mathfrak{C})$-problem, has been studied. The problem input consists of a graph $G=(V, E)$ and two weight functions for the edges: the length $l: E \rightarrow \mathbb{R}_{+}$and the cost $c: E \rightarrow \mathbb{R}_{+}$. $A$ is a criterion on the weight function $c$ and $B$ is a criterion on the weight function $l$. For instance, $A$ could be cost and $B$ could be diam (as defined in section 2). $\lambda$ is a real number not less than 1 and $\mathfrak{C}$ is a class of graphs that limits the possible solutions (e.g., trees) [Bálint 2003].

Let $\mathfrak{C}(G)$ denote the class of all spanning subgraphs of $G$ belonging to the class $\mathfrak{C}$, i.e., $\mathfrak{C}(G):=\{S \in \mathfrak{C} \mid S$ is a spanning subgraph of $G\}$. The minimum value of criterion $A$ is defined as $A^{*}=\min \{A(S) \mid S \in \mathfrak{C}(G)\}$. The minimum value of criterion $B$ under a budget on $A$ is defined as $B_{\lambda}=\min \left\{B(S) \mid S \in \mathfrak{C}(G), A(S) \leq \lambda A^{*}\right\}$. Note that $\lambda$ indirectly defines a budget [Bálint 2003].

A solution to the $(A, B, \lambda, \mathfrak{C})$-problem is a spanning subgraph $S \in \mathfrak{C}(G)$ such that $A(S) \leq \lambda A^{*}$ and $B(S)=B_{\lambda}$. As an example, the MSDP, defined in Section 2, is almost equivalent to the (cost, diam, $\lambda$, spanning subgraph)-problem. The only difference is that here the budget is set indirectly by $\lambda$ instead of directly by $R$ on the MSDP, i.e., for a budget $R$ we should set $\lambda=R / A^{*}$.

An $(\alpha, \beta)$-approximation to the $(A, B, \lambda, \mathfrak{C})$-problem is a spanning subgraph $S \in$ $\mathfrak{C}(G)$ such that $A(S) \leq \alpha \lambda A^{*}$ and $B(S) \leq \beta B_{\lambda}$ [Bálint 2003].

It has been proved that, unless $P=N P$, for any constant $\beta$ there is no polynomial time algorithm to solve the $(1, \beta)$-approximation to the (cost, diam, 1 , spanning tree)problem, even if both criteria are under the same cost function $c: E \rightarrow\{1,2\}$ [Bálint 2003]. In other words, there is no polynomial time algorithm to find a minimum spanning tree (since $\lambda=\alpha=1$ ) with at most $\beta$ times the diameter of any minimum spanning tree. We shall use this result to prove that there is no polynomial time algorithm to solve the $\beta$-approximation to the MSDP for any $\beta$ constant. 


\section{Our results}

Suppose that for some constant $\beta$ there is a polynomial time $\beta$-approximation algorithm for the MSDP. Given an instance $I$ of the (cost, diam, 1 , spanning tree)-problem, we can create an instance $I^{\prime}$ of the MSDP by using the same graph, weight functions and setting the budget $R=\operatorname{cost}(\operatorname{MST}(G))$, i.e., the cost of a minimum spanning tree for $G$ (since $\lambda=1)$.

If we solve the MSDP for $I^{\prime}$, the result might not be a tree, due to the possibility of zero cost edges in the solution. Then, there would be no trivial way to transform this solution back to one for the (cost, diam, 1, spanning tree)-problem, since removing any edges forfeits our guarantees on the diameter. In this case, we would not be able to translate the lower bound for spanning trees from Bálint to a lower bound for general spanning subgraphs. However, we can use the stronger lower bound by Bálint which holds even when both criteria are under the same cost function $c: E \rightarrow\{1,2\}$.

Thus, assuming that $I$ only has edges with costs 1 or 2 , we create $I^{\prime}$ as before and then we solve the MSDP for $I^{\prime}$. Since there are no zero cost edges, the solution found would be a minimum spanning tree with diameter at most $\beta$ times that of any other minimum spanning tree.

The minimum spanning tree found would be a $(1, \beta)$-approximation to the (cost, diam, 1 , spanning tree)-problem, contradicting the result by Bálint. Therefore, there is no polynomial time $\beta$-approximation algorithm for the MSDP for any constant $\beta$, unless $P=N P$.

\section{Future work}

Our result cuts short any effort to find a $\beta$-approximation for the MSDP. Nonetheless, a promising line of research is to investigate $(\alpha, \beta)$-approximation algorithms for the MSDP.

\section{References}

Bálint, V. (2003). The non-approximability of bicriteria network design problems. Journal of Discrete Algorithms, 1:339-355.

Papadimitriou, C. H. and Steiglitz, K. (1998). Combinatorial Optimization: Algorithms and Complexity. Courier Corporation.

Plesnik, J. (1981). The complexity of designing a network with minimum diameter. Networks, 11:77-85.

Trevisan, L. (2014). Inapproximability of Combinatorial Optimization Problems. In: Paradigms of Combinatorial Optimization, chapter 13, pages 381-434. WileyBlackwell.

Williamson, D. P. and Shmoys, D. B. (2011). The Design of Approximation Algorithms. Cambridge University Press, New York, NY, USA, 1st edition. 